\title{
Space-time variations of megazoobenthos subject to natural and anthropogenic impacts in two Cuban bays: evidence of recovery?
}

\author{
Alexander Lopeztegui-Castillo ${ }^{1,2}$, Norberto Capetillo-Piñar ${ }^{3}$, Abel Betanzos-Vega ${ }^{1}$ \\ Beatriz Martínez-Daranas ${ }^{4}$, Diana Martínez-Coello ${ }^{1}$ \& Leonardo Andrés Abitia-Cárdenas ${ }^{2}$ \\ ${ }^{1}$ Centro de Investigaciones Pesqueras, Playa, La Habana, Cuba \\ ${ }^{2}$ Instituto Politécnico Nacional, Centro Interdisciplinario de Ciencias Marinas, La Paz, BCS, México \\ ${ }^{3}$ Federación de Cooperativas Pesqueras Zona Centro, La Paz, BCS, México \\ ${ }^{4}$ Centro de Investigaciones Marinas, Universidad de La Habana, Miramar, La Habana, Cuba \\ Corresponding author: Leonardo Andrés Abitia-Cárdenas (labitia@ipn.mx)
}

\begin{abstract}
The spatiotemporal variations of megazoobenthos on soft bottoms in Buena Vista (BV) and San Juan de Los Remedios (SJR) bays, Cuba, were assessed to infer the effects of the elimination of bottom trawl fisheries and the creation of protected areas. Data were collected at 11 sites in 2010-2013 (six routines and five exploratories) and 11 sites in 2014-2017 (four routines and seven exploratories). Samples were obtained by dredging. Mollusks were the most frequent and diverse group, followed by echinoderms and crustaceans. Total density $\left(7.60\right.$ ind $\left.\mathrm{m}^{-2}\right)$ and biomass $\left(15.82 \mathrm{~g} \mathrm{~m}^{-2}\right.$ ) of the benthic community were highest in SJR in 2015. Although total biomass was lower at BV $(\mathrm{t}=2.21 ; P=0.032)$, there were no significant differences in density between bays $(\mathrm{t}=0.77 ; P=0.444)$. The site with a lower total density and biomass was located at $\mathrm{BV}$. There were no differences between years except at one site, where density increased between 2010 and 2017. Total density and biomass were not significantly different inside and outside of the Zone Under Special Regime of Use and Protection (ZUSRUP) at the beginning of the sampled period. However, both density and biomass were significantly higher within the ZUSRUP at the end (2017), which suggests this protected area's effectivity. The progressive increase in density at one site and greater density and biomass values at SJR, where bottom trawl fisheries were more intensive, suggest eliminating bottom trawl nets may have contributed to the benthic fauna recovery.
\end{abstract}

Keywords: megazoobenthos; trawl fishing; habitat quality; environmental impact; protected areas

\section{INTRODUCTION}

Marine benthos constitutes an important element in understanding trophic relationships among species, particularly those inhabiting areas over continental platforms, where they can be used to infer the productive potential of a given fishing area (Gómez et al. 1980, Lalana et al. 1989, Herrera et al. 1991, Alcolado et al. 1998). Benthic communities contribute to ecosystem functioning by decomposing organic matter, regenerating nutrients, and providing higher trophic food levels (Middelburg 2018). Another useful characteristic of benthos is that it can be used as an indicator of environmental quality, based mainly on estimates of diversity, abundance, and biomass (Alcolado et al. 1998, Chirwa \& Chilim 2017, Matangulu et al. 2017, Ovaskainen et al. 2019), on the calculation of ecological indices -such as species richness (S), Shannon-Weaver diversity (H'), Azti marine biotic index (AMBI), trophic index (TI), Turkish benthic index (TUBI) and biological monitoring working party (BMWP), among others (Çinar et al. 2015, Castellanos-Romero et al. 2017, Fouad et al. 2019), and on the observation of particular species used as bioindicators or biomonitors (Mohammad-Karami et al. 2014, Munroe et al. 2018, Tlili \& Mouneyrac 2019).

Within the framework of the GEF/PNUD SabanaCamaguiey project, which focuses on potentiating biodiversity conservation, environmental conditions in the bays and ecosystems found in the SabanaCamagüey Archipelago (SCA), located in the centraleastern area of the northern Cuban coast, were studied. Between 2010 and 2013, this project involved the use

Corresponding editor: Laura Schejter 
of soft-bottom benthic communities of the Buena Vista (BV) and San Juan de Los Remedios (SJR) bays to infer the degree of habitat recovery after the implementation of management measures focused on creating protected areas, since 2009, and eliminating the use of bottom trawls, since 2003. Both bays constitute feeding habitats for several commercial interest species, such as the Caribbean spiny lobster Panulirus argus (Latreille 1804), which is considered the central fishery resource of Cuba (Cruz et al. 1990, Puga et al. 2018). Changes in benthic communities due to natural or anthropogenic causes can alter the ecosystem functioning, including carbon pathways, also affecting the food availability for $P$. argus, which, along with low water quality, could affect the abundance, distribution, and physiological condition of lobsters (Martínez-Coello et al. 2015, McGovern et al. 2020).

The development of tourism in the SCA has led to different stress levels over the submarine platform and cays, which has led to biodiversity decreasing along with climate change effects. Extreme meteorological events are among the most important natural factors disturbing the area (Fernández-Vila \& Chirino-Núñez 1993, Betanzos-Vega et al. 2013). The region has been directly or indirectly affected by 14 hurricanes of great intensity between 2001 and 2016. However, the most massive and most recent impact was represented by hurricane Irma in September 2017, which crossed the entire archipelago as categories 5 and 3 on the SaffirSimpson scale.

Among the anthropogenic factors of most significant influence, organic contamination, eutrophication due to agriculture, and lack of residual treatment have been reported (Alcolado et al. 1999, Montalvo et al. 2004), as well as the destructive effects of trawl nets, the damming of rivers, and the construction of hotels and causeways (Alcolado et al. 2007, MartínezDaranas \& Suárez 2018). The shallowness of the area leads to increases in temperature and evaporation rates, which, along with scarcity in freshwater sources, gives rise to elevated salinity values (Fernández-Vila \& Chirino-Núñez 1993). Benthic community, driven by river influence on sediment grain size and high rates of sediment deposition, leads to a decrease in taxonomic and functional diversity indices, including the loss of functional traits (i.e. downward conveyors, epifauna) with potential implications for benthic ecosystem functioning (McGovern et al. 2020). This effect is aggravated by construction works that limit water circulation (Betanzos-Vega et al. 2010).

There are no historical records that would allow exhaustive comparisons of the benthic communities found on this archipelago's soft bottoms. At the end of the past century, it was determined that the mesobenthic fauna (between 2-0.5 mm) was dominated by foraminiferans and nematodes (Jiménez \& Ibarzábal 1982). Regarding fauna $>4 \mathrm{~mm}$ (megazoobenthos), Alcolado et al. (1998) reported an average species richness of 45 per site, with the highest values (72) and dominance of gastropods towards the edge of the platform. During the first stage of the GEF/PNUD project, 447 species were reported over the SCA's soft bottoms (Alcolado et al. 1999). During the second stage, 598 species were reported (Alcolado et al. 2007), but no malacofauna analyses were performed. The malacofauna constitutes the main food for $P$. argus (Espinosa et al. 1990, Herrera et al. 1991, MartínezCoello et al. 2015). It is considered a fundamental bioindicator group (Alcolado et al. 1998, Tlili \& Mouneyrac 2019).

In this context, the present study's objective was to assess megazoobenthos spatiotemporal variations over soft bottoms in the SCA's north-central sector (bays of Buena Vista and San Juan de Los Remedios). With an emphasis on the community of shelled mollusks, to assess the effect of management measures such as eliminating bottom trawl fishing and creating protected areas.

\section{MATERIALS AND METHODS}

\section{Study area}

The SCA covers $465 \mathrm{~km}$ between Punta Hicacos and Bahía de Nuevitas. It comprises approximately 2517 cays ( $60 \%$ of all Cuban cays) and has an average depth of $3 \mathrm{~m}$. Seagrasses, mainly Thalassia testudinum K.D. Koenig 1805 , with medium or low density, cover $76 \%$ of the soft bottom in this region (Alcolado et al. 2007). The study area comprised soft-bottom areas on either side of the causeway that unites Cuba's island to Cayo Santa Maria. It included the eastern side of San Juan de Los Remedios Bay (SJR) and the western side of Buena Vista Bay (BV). The average depth in these areas ranges between $2.06(\mathrm{BV})$ and $2.19 \mathrm{~m}(\mathrm{SJR})$, although depths can reach up to $4 \mathrm{~m}$ in the central part of the bays, with short stretches of up to $6 \mathrm{~m}$ mainly in oceanic waters exchange areas (Betanzos-Vega et al. 2013).

Most sampled sites were located among the cays at the narrow platform's northern limit (Fig. 1). Some sites were situated within the Zone Under Special Regime of Use and Protection (ZUSRUP), created in 2009 (Resolution 478/09). Other characteristics of the sampled sites are given in Table 1. In the studied area, bottom trawl fisheries had been banned for two miles from the causeway since 2003 (Resolution 233/03). Inside the ZUSRUP, the use of any type of seine net was banned since its creation in 2009. Anywhere outside the ZUSRUP bottom trawl fisheries were banned since 2012 (Resolution 503/12). 


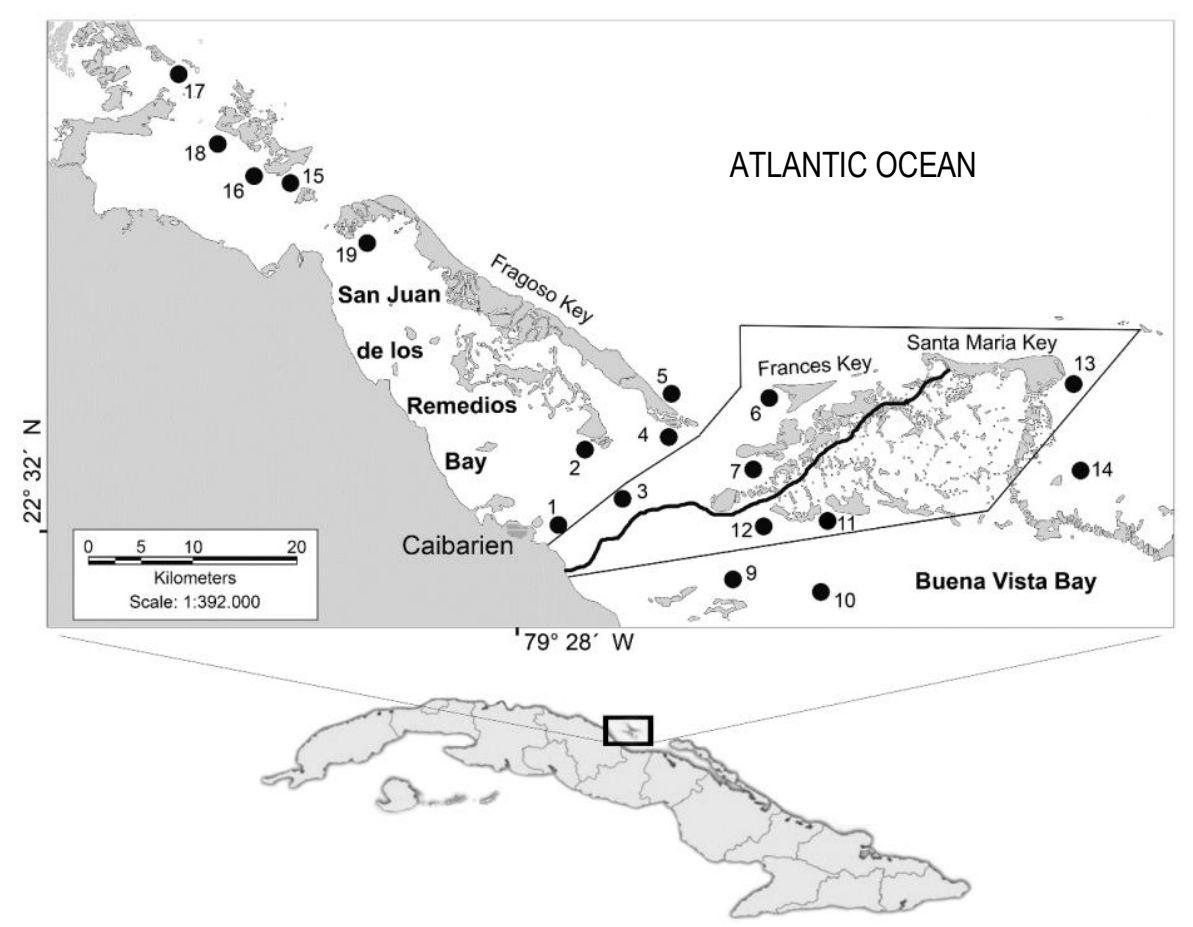

Figure 1. Study zone. San Juan de Los Remedios and Buena Vista bays in Sabana-Camagüey Archipelago, Cuba. Sites from 1 to 12 were sampled in the 2010-2013 period (PNUD Project). Sites 9-12 were resampled until 2017. The continuous straight lines delimit that area currently considered Zone Under Special Regime of Use and Protection (ZUSRUP); the curved line represents the causeway.

\section{Biological sampling and statistical analysis}

Samples for benthic fauna analysis were obtained by dredging with a rectangular device of $54 \times 38 \mathrm{~cm}^{2}$ effective area and $4 \mathrm{~mm}$ mesh attached. Three replicates (dredges) per site were attained using a $14 \mathrm{~m}$ length Lambda ship, moving at a constant speed of $2 \mathrm{kn}$ for $40 \mathrm{~s}$ on average, meaning a total sampled area of 21 $\mathrm{m}^{2}$ per dredge. The provided value of each site was an average of the three dredges. After dredging, alive organisms were collected from a sieve of the same size $(4 \mathrm{~mm})$ and stored in plastic bags with buffered $4 \%$ formaldehyde. In the laboratory, organisms were identified under a stereoscopic microscope, helped by specialized researchers.

Taxonomic identification of mollusks, a group that was analyzed in detail due to their importance as bioindicators and as a food source for Panulirus argus (Espinosa et al. 1990, Herrera et al. 1991, Alcolado \& Espinosa 1996), was carried out based on publications by Warmke \& Abbott (1975), Mikkelsen \& Bieler (2007), and Redfern (2013). The remaining organisms were identified only to the level of large taxonomic groups, taking into account basic literature, catalogs, and species lists given for Cuban waters (Ibarzábal et al. 1999, Lalana \& Ortiz 2000, Ortiz \& Lalana 2001, Del Valle et al. 2005, Claro 2007).

Organisms were counted (n), weighed, and grouped into broad taxonomic categories. The percentage of frequency of occurrence $(\mathrm{FO}=$ number of sites in which organism was found / total number of sites $\times 100$ ) was calculated. The most frequently recorded species and the species that provided most inputs to biomass were identified. The constancy index (CI) was calculated for shell mollusks following criteria by Prieto et al (2003):

$$
\mathrm{CI}=\mathrm{Ri} \times 100 / \mathrm{R}
$$

where $R_{i}$ is the number of samples (replicates) in which species $i$ appears, and $\mathrm{R}$ is the total number of samples.

Statistical analyses were performed using Statistica 7.0. Multiple comparisons of abundance and biomass were carried out with a unifactorial ANOVA, using Tukey's LSD post-hoc test to detect differences between means. For paired comparisons (between bays or between locations), Student's $t$-tests for independent groups were used. The significance level used was $P=$ 0.05 . 
Table 1. Sampled sites. SJR: San Juan de Los Remedios Bay, BV: Buena Vista Bay, I: inside the protected area, O: outside the protected area, E: exploratory (sampled once or twice), R: routine (sampled more than twice), Ext: external (near to the platform edges), Int: internal (near to the coast or in the middle of the bays).

\begin{tabular}{|c|c|c|c|c|c|c|c|}
\hline Site & Coordinates & $\begin{array}{l}\text { Depth } \\
\text { (m) }\end{array}$ & SJR/BV & $\mathrm{I} / \mathrm{O}$ & $\begin{array}{c}\text { Sampling } \\
\text { years }\end{array}$ & $\mathrm{E} / \mathrm{R}$ & Int/Ext \\
\hline 1 & $22^{\circ} 31^{\prime} 50^{\prime \prime} \mathrm{N}, 79^{\circ} 25^{\prime} 40^{\prime \prime} \mathrm{W}$ & 1.4 & SJR & $\mathrm{O}$ & 2 & $\mathrm{E}$ & Int \\
\hline 2 & $22^{\circ} 35^{\prime} 37^{\prime \prime N}, 79^{\circ} 24^{\prime} 13^{\prime \prime} \mathrm{W}$ & 2.1 & SJR & $\mathrm{O}$ & 2 & $\mathrm{E}$ & Int \\
\hline 3 & $22^{\circ} 32^{\prime} 49^{\prime \prime} \mathrm{N}, 79^{\circ} 21^{\prime} 43^{\prime \prime} \mathrm{W}$ & 2.8 & SJR & $\mathrm{I}$ & 3 & $\mathrm{R}$ & Int \\
\hline 4 & $22^{\circ} 36^{\prime} 10^{\prime \prime} \mathrm{N}, 79^{\circ} 19^{\prime} 35^{\prime \prime} \mathrm{W}$ & 2.3 & SJR & $\mathrm{O}$ & 3 & $\mathrm{R}$ & Ext \\
\hline 5 & $22^{\circ} 38^{\prime} 01^{\prime \prime N}, 79^{\circ} 19^{\prime} 29^{\prime \prime} \mathrm{W}$ & 2.9 & Out of bay & $\mathrm{O}$ & 2 & $\mathrm{E}$ & Ext \\
\hline 6 & $22^{\circ} 38^{\prime} 14^{\prime \prime N}, 79^{\circ} 13^{\prime} 45^{\prime \prime} \mathrm{W}$ & 3.3 & Out of bay & $\mathrm{I}$ & 2 & $\mathrm{E}$ & Ext \\
\hline 7 & $22^{\circ} 34^{\prime} 21^{\prime \prime} \mathrm{N}, 79^{\circ} 15^{\prime} 10^{\prime \prime} \mathrm{W}$ & 1.9 & SJR & I & 3 & $\mathrm{R}$ & Int \\
\hline 9 & $22^{\circ} 28^{\prime} 11^{\prime \prime N}, 79^{\circ} 16^{\prime} 26^{\prime \prime} \mathrm{W}$ & 3.1 & $\mathrm{BV}$ & $\mathrm{O}$ & 5 & $\mathrm{R}$ & Int \\
\hline 10 & $22^{\circ} 27^{\prime} 30^{\prime \prime} \mathrm{N}, 79^{\circ} 11^{\prime} 40^{\prime \prime} \mathrm{W}$ & 3.3 & $\mathrm{BV}$ & $\mathrm{O}$ & 5 & $\mathrm{R}$ & Int \\
\hline 11 & $22^{\circ} 31^{\prime} 40^{\prime \prime} \mathrm{N}, 79^{\circ} 10^{\prime} 46^{\prime \prime} \mathrm{W}$ & 1.5 & BV & I & 5 & $\mathrm{R}$ & Int \\
\hline 12 & $22^{\circ} 31^{\prime} 26^{\prime \prime} \mathrm{N}, 79^{\circ} 14^{\prime} 24^{\prime \prime} \mathrm{W}$ & 3.0 & $\mathrm{BV}$ & I & 5 & $\mathrm{R}$ & Int \\
\hline 13 & $22^{\circ} 38^{\prime} 41^{\prime \prime N}, 78^{\circ} 57^{\prime} 06^{\prime \prime} \mathrm{W}$ & 2.8 & Out of bay & I & 1 & $\mathrm{E}$ & Ext \\
\hline 14 & $22^{\circ} 34^{\prime} 08^{\prime \prime} \mathrm{N}, 78^{\circ} 56^{\prime} 54^{\prime \prime} \mathrm{W}$ & 3.8 & Out of bay & $\mathrm{O}$ & 1 & $\mathrm{E}$ & Ext \\
\hline 15 & $22^{\circ} 49^{\prime} 28^{\prime \prime} \mathrm{N}, 79^{\circ} 40^{\prime} 13^{\prime \prime} \mathrm{W}$ & 2.9 & SJR & $\mathrm{O}$ & 1 & $\mathrm{E}$ & Ext \\
\hline 16 & $22^{\circ} 49^{\prime} 35^{\prime \prime} \mathrm{N}, 79^{\circ} 42^{\prime} 13^{\prime \prime} \mathrm{W}$ & 2.5 & SJR & $\mathrm{O}$ & 1 & $\mathrm{E}$ & Int \\
\hline 17 & $22^{\circ} 55^{\prime} 08^{\prime \prime} \mathrm{N}, 79^{\circ} 46^{\prime} 43^{\prime \prime} \mathrm{W}$ & 2.1 & SJR & $\mathrm{O}$ & 1 & $\mathrm{E}$ & Ext \\
\hline 18 & $22^{\circ} 51^{\prime} 44^{\prime \prime N}, 79^{\circ} 44^{\prime} 34^{\prime \prime} \mathrm{W}$ & 2.5 & SJR & $\mathrm{O}$ & 1 & $\mathrm{E}$ & Int \\
\hline 19 & $22^{\circ} 46^{\prime} 12^{\prime \prime N}, 79^{\circ} 36^{\prime} 30^{\prime \prime} \mathrm{W}$ & 2.3 & SJR & $\mathrm{O}$ & 1 & $\mathrm{E}$ & Int \\
\hline
\end{tabular}

\section{RESULTS}

\section{Taxonomic composition of the megazoobenthic community}

Organisms found in the 2010-2013 (GEF/PNUD project) were grouped into 14 taxonomic groups, which were also recorded during the later sampling (Table 2). Megazoobenthic communities were composed by 340 species: 93 Gastropoda, 84 Bivalvia, 3 Scaphopoda, 5 Polyplacophora, 26 Brachyura, 31 Anomura, 24 Caridea, 12 Isopoda, 10 Stomatopoda, 20 Ophiuroidea, 10 Echinoidea, 2 Ascidiacea, 10 Polychaeta and 9 Porifera. The highest species richness (total $\mathrm{S}=141$, considering all the sampled sites) was obtained in 2015; however, the average value per site for all sampled period was $S=17(S=21$ per site in $S J R$ and $S=13$ per site in $\mathrm{BV}$ ).

Mollusks constituted $54.7 \%$ of species and were the most frequently recorded group during 2010-2017. Among them, gastropods were the most frequently recorded organisms during all years, followed by ophiuroids, bivalves, and a lower proportion of crustaceans such as anomurans and caridean shrimps (Table 2). During all sampled periods (2010-2017), 186 species of shell mollusks were identified in the soft bottoms of San Juan de Los Remedios and Buena Vista bays, Sabana-Camagüey Archipelago, Cuba (Table 3).

The most frequently recorded gastropods were Cerithium eburneum Bruguière 1792, Prunum apici- num (Menke 1828), Modulus modulus (Linnaeus 1758), Phrontis alba (Say 1826), and Bulla striata Bruguière 1792. At site 12, where seagrasses were abundant, the herbivorous species Tegula fasciata (Born 1778) was dominant. The most frequently recorded bivalves included Chione cancellata (Linnaeus 1767) (also dominant at sites 1 and 3), Dallocardia muricata (Linnaeus 1758), Argopecten gibbus (Linnaeus 1758), Pitar simpsoni (Dall 1895), and Laevicardium mortoni (Conrad 1831). Gastropods dominated (higher FO) the area except at site 18, at which the bivalves Serratina martinicensis (d'Orbigny 1853), Caryocorbula swiftiana (C.B. Adams 1852), and Macoma tenta (Say 1834) were better represented.

Among ophiuroids, Ophiotrix sp. was the bestrepresented genera, mainly the species Ophiothrix angulata (Say 1825). The hermit crabs (anomurans) and non-commercial shrimps (carideans) were the most frequently recorded crustaceans. Anomurans dominated at external SJR sites, but isopods and decapods were dominant in the interior SJR sites and western BV. Ophiuroids were the most common echinoderms, but the green sea urchin Lytechinus variegatus (Lamarck 1816) was dominant in biomass. Nevertheless, at some sites located on the platform edge (sites 5 and 6), numerous sea biscuit Clypeaster rosaceus (Linnaeus 1758) were found. Chondrilla nucula Schmidt 1862, was the best-represented sponge, registering a high frequency of occurrence and biomass at interior sites. 
Table 2. Number of organisms (n), number of species (S), and frequency of occurrence (FO) in \% of the taxonomic groups found as megazoobenthos (>4 mm) in the soft bottoms of San Juan de Los Remedios and Buena Vista bays.

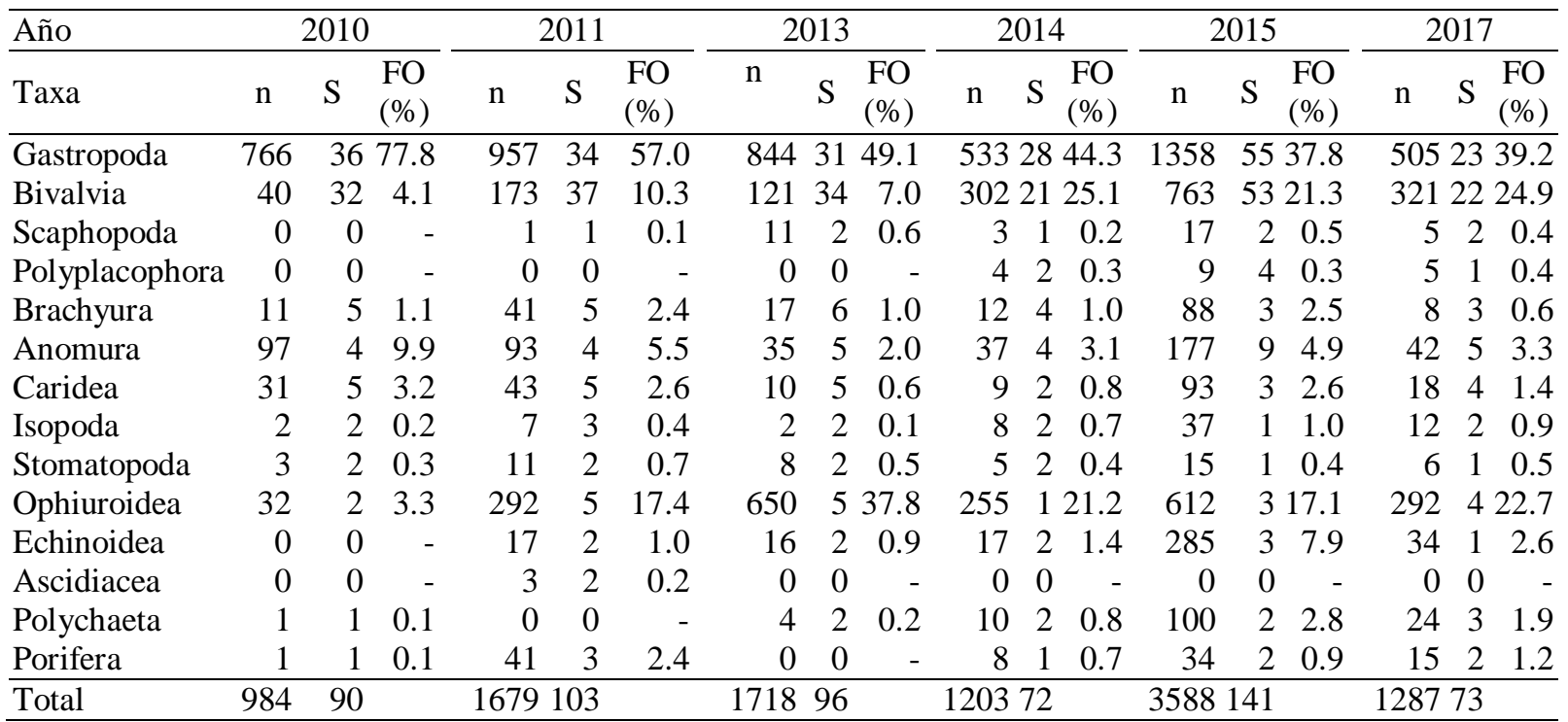

\section{Megazoobenthos density and biomass in the 2010- 2013 period}

The analysis of abundance for 2010-2013 (routine sites of this period: $3,4,9,7,10$, and 12) showed that site 10 (at BV) presented the lowest total density $\left(0.16\right.$ ind. $\left.\mathrm{m}^{-2}\right)$ of organisms. General averages did not show significant differences among years $(P=0.345)$. However, there were significant differences among sites $(P=$ $0.001)$ and in the site-year interaction $\left[\mathrm{F}_{(10 ; 36)}=2.94, P\right.$ $=0.008]$. Interannual variations at each site showed that the trend was not the same in all cases. A gradual total density increase was recorded only at site 12 (Fig. 2a). The annual average of biomass at the same sites $(3,4$, $9,7,10$, and 12) did not show similar variations among sites. There was a slight but gradual increase only at site 7. Averages biomass values were not statistically different among years $(P=0.055)$, but among sites $(P$ $=0.001)$ and in the site-year interaction $\left[\mathrm{F}_{(10 ; 36)}=4.37\right.$, $P=0.001]$. The lowest total biomass was recorded at site 10 , with an average value of $0.04 \mathrm{~g} \mathrm{~m}^{-2}$ (Fig. 2b). The comparison of annual average values in 2010-2013 period showed that total density $\left[\mathrm{F}_{(2 ; 51)}=0.62, P=\right.$ $0.544]$, as well as total biomass $\left[\mathrm{F}_{(2 ; 51)}=1.40, P=\right.$ 0.257], were similar among these years.

Taking into account the 11 sites sampled in 20112013 , it was found that density $\left[\mathrm{F}_{(10 ; 22)}=1.72, P=\right.$ $0.139]$ and biomass $\left[\mathrm{F}_{(10 ; 2)}=1.09, P=0.409\right]$ did not vary significantly between sites in 2011. In 2013 significant differences between sites in density $\left[\mathrm{F}_{(10 ; 2)}\right.$
$=7.66, P=0.001]$ and in biomass $\left[\mathrm{F}_{(10 ; 22)}=6.68, P=\right.$ 0.001 ] due to the greater values at site 11 were found.

\section{Megazoobenthos density and biomass in all sampled periods}

Comparison between bays and between protected and non-protected areas

Density $\left(7.60\right.$ ind $\left.\mathrm{m}^{-2}\right)$ and biomass $\left(15.81 \mathrm{~g} \mathrm{~m}^{-2}\right)$ values were highest in 2015 . The comparison between bays from 2010 to 2015 did not show differences $(\mathrm{t}=0.77$, $P=0.444$ ) in the values of total density of organisms, but total biomass was significantly lower $(\mathrm{t}=2.21, P=$ 0.032) at BV (Fig. 3).

No significant differences in total density and total biomass of organisms were found among sites located inside and outside the ZUSRUP at the beginning of the sampled period (2011). However, at the end of the sampled period (2017), the two variables were significantly higher at sites within the ZUSRUP (Table 4).

The comparative analysis undertaken for sites 9,10 and 12 , sampled regularly during five years of the study (2010, 2011, 2013, 2014, and 2017), showed that there were no significant differences among the annual average values of density $\left[\mathrm{F}_{(4 ; 40)}=0.45, P=0.769\right]$ or biomass $\left[\mathrm{F}_{(4 ; 40)}=2.03, P=0.108\right]$ over time (Fig. 4). A similar analysis performed for the site 11 showed significant differences between years for density of organisms $\left[\mathrm{F}_{(4 ; 10)}=8.58, P=0.003\right]$, but not for biomass $\left[\mathrm{F}_{(4 ; 10)}=2.15, P=0.148\right]$ (Fig. 5). 
Table 3. Alphabetical list of megazoobenthic ( $>4 \mathrm{~mm}$ ) shell mollusks found in the soft bottoms of San Juan de Los Remedios and Buena Vista bays, in 2010-2017 period. CI: constancy index.

\begin{tabular}{|c|c|c|c|}
\hline Gastropoda & $\mathrm{CI}$ & Bivalvia & $\mathrm{CI}$ \\
\hline$\overline{\text { Atlantilux exigua (C.B. Adams 1845) }}$ & 6.8 & Acorylus gouldii (Hanley 1846) & 9.1 \\
\hline Bailya parva (C.B. Adams 1850) & 6.8 & Aequipecten exasperatus (G.B. Sowerby II 1842) & 25.0 \\
\hline Bostrycapulus aculeatus (Gmelin 1791) & 38.6 & Aequipecten muscosus (W. Wood 1828) & 40.9 \\
\hline Bulla striata Bruguière 1792 & 81.8 & Americardia guppyi (Thiele 1910) & 22.7 \\
\hline Calliostoma pulchrum (C.B. Adams 1850) & 6.8 & Americardia media (Linnaeus 1758) & 6.8 \\
\hline Cassis flammea (Linnaeus 1758) & 4.5 & Ameritella consobrina (d'Orbigny 1853) & 18.2 \\
\hline Cassis madagascariensis Lamarck 1822 & 4.5 & Anadara notabilis (Röding 1798) & 25.0 \\
\hline Cassis tuberosa (Linnaeus 1758) & 4.5 & Anomalocardia flexuosa (Linnaeus 1767) & 4.5 \\
\hline Cerithium atratum (Born 1778) & 6.8 & Anomalocardia puella (Pfeiffer in Philippi 1846) & 4.5 \\
\hline Cerithium eburneum Bruguière 1792 & 93.2 & Anomia peruviana d'Orbigny 1846 & 36.4 \\
\hline Cerithium litteratum (Born 1778) & 6.8 & Antillipecten antillarum (Récluz 1853) & 25.0 \\
\hline Cerithium lutosum Menke 1828 & 9.1 & Arcopsis adamsi (Dall 1886) & 15.9 \\
\hline Cerithium muscarum Say 1832 & 40.9 & Argopecten gibbus (Linnaeus 1758) & 68.2 \\
\hline Cerodrillia thea (Dall 1884) & 25.0 & Atrina rigida (Lightfoot 1786$)$ & 6.8 \\
\hline Charonia variegata (Lamarck 1816) & 4.5 & Barbatia candida (Heilblind, 1779) & 4.5 \\
\hline Chicoreus florifer (Reeve 1846) & 38.6 & Barbatia domingensis (Lamarck 1819) & 18.2 \\
\hline Clathrodrillia solida (C.B. Adams 1850) & 2.3 & Caryocorbula swiftiana (C.B. Adams 1852) & 25.0 \\
\hline Columbella mercatoria (Linnaeus 1758) & 25.0 & Chama macerophylla Gmelin 1791 & 4.5 \\
\hline Columbella rusticoides Heilprin 1886 & 27.3 & Chama sarda Reeve 1847 & 4.5 \\
\hline Conasprella jaspidea (Gmelin 1791) & 15.9 & Chione cancellata (Linnaeus 1767) & 86.4 \\
\hline Conasprella mindana (Hwass in Bruguière 1792) & 6.8 & Chione elevata (Say 1822) & 72.7 \\
\hline Costoanachis sparsa (Reeve 1859) & 31.8 & Clathrolucina costata (d'Orbigny 1845) & 9.1 \\
\hline Crassispira cubana (Melvill 1923) & 6.8 & Codakia orbicularis (Linnaeus 1758) & 11.4 \\
\hline Crassispira fuscescens (Reeve 1843) & 27.3 & Caryocorbula contracta (Say 1822) & 22.7 \\
\hline Crepidula navicula (Mörch 1877) & 47.7 & Crassinella lunulata (Conrad 1834) & 27.3 \\
\hline Cycloscala echinaticosta (d'Orbigny 1842) & 6.8 & Crassinella martinicensis (d'Orbigny 1853) & 4.5 \\
\hline Cylindrobulla beauii P. Fischer 1857 & 27.3 & Crenella divaricata (d'Orbigny 1853) & 2.3 \\
\hline Diodora arcuata (G.B. Sowerby II 1862) & 2.3 & Ctena orbiculata (Montagu 1808) & 25.0 \\
\hline Diodora cayenensis (Lamarck 1822) & 6.8 & Cumingia lamellosa G.B. Sowerby I 1833 & 2.3 \\
\hline Diodora jaumei Aguayo \& Rehder 1936 & 4.5 & Cumingia tellinoides (Conrad 1831) & 27.3 \\
\hline Diodora minuta (Lamarck 1822) & 4.5 & Dallocardia muricata (Linnaeus 1758) & 70.5 \\
\hline Eulithidium adamsi (Philippi 1853) & 6.8 & Diplodonta nucleiformis (W. Wagner 1840) & 6.8 \\
\hline Eulithidium thalassicola (R. Robertson 1958) & 9.1 & Diplodonta punctata (Say 1822) & 9.1 \\
\hline Eupleura sulcidentata Dall 1890 & 6.8 & Divaricella dentata $($ Wood 1815$)$ & 6.8 \\
\hline Eoacmaea pustulata (Helbling 1779) & 9.1 & Divalinga quadrisulcata (d'Orbigny 1845) & 6.8 \\
\hline Falsuszafrona idalina (Duclos 1840) & 4.5 & Dosinia concentrica (Born 1778) & 4.5 \\
\hline Falsuszafrona pulchella (Blainville 1829) & 15.9 & Entodesma brasiliense (Gould 1850) & 15.9 \\
\hline Falsuszafrona dicomata (Dall 1889) & 6.8 & Eurytellina alternata (Say 1822) & 9.1 \\
\hline Fasciolaria tulipa (Linnaeus 1758) & 31.8 & Eurytellina angulosa (Gmelin 1791) & 9.1 \\
\hline Fenimorea fucata (Reeve 1845) & 25.0 & Eurytellina lineata (W. Turton 1819) & 9.1 \\
\hline Gibberula fluctuata (C.B. Adams 1850) & 2.3 & Eurytellina nitens (C.B. Adams 1845) & 20.5 \\
\hline Gutturnium muricinum (Röding 1798) & 15.9 & Fugleria tenera (C.B. Adams 1845) & 4.5 \\
\hline Haminoea antillarum (d'Orbigny 1841) & 9.1 & Fulvia laevigata (Linnaeus 1758) & 43.2 \\
\hline Haminoea elegans (Gray 1825) & 6.8 & Glycymeris marmorata (Linnaeus 1758) & 6.8 \\
\hline Hesperisternia multangulus (Philippi 1848) & 15.9 & Gouldia cerina (C.B. Adams 1845) & 15.9 \\
\hline Leucozonia nassa (Gmelin 1791) & 4.5 & Laciolina magna (Spengler 1798) & 9.1 \\
\hline Lithopoma phoebium (Röding 1798) & 6.8 & Laevicardium mortoni (Conrad 1831) & 63.6 \\
\hline Lobatus costatus (Gmelin 1791) & 4.5 & Leiosolenus bisulcatus (d'Orbigny 1853) & 2.3 \\
\hline Lobatus gigas (Linnaeus 1758) & 11.4 & Leukoma granulata (Gmelin 1791) & 2.3 \\
\hline Lobatus raninus (Gmelin 1791) & 2.3 & Limaria pellucida (C.B. Adams 1848) & 13.6 \\
\hline Lucapina suffusa (Reeve 1850) & 13.6 & Lucina pensylvanica (Linnaeus 1758) & 38.6 \\
\hline Macrocypraea zebra (Linnaeus 1758) & 2.3 & Lucinisca muricata (Spengler 1798) & 59.1 \\
\hline Mitrella dichroa (G.B. Sowerby I 1844) & 6.8 & Macoploma tageliformis (Dall 1900) & 6.8 \\
\hline
\end{tabular}


continuation

\begin{tabular}{|c|c|c|c|}
\hline Gastropoda & $\mathrm{CI}$ & Bivalvia & $\mathrm{CI}$ \\
\hline Mitrella ocellata (Gmelin 1791) & 2.3 & Macoploma tenta (Say 1834) & 59.1 \\
\hline Modulus modulus (Linnaeus 1758) & 90.9 & Mactrotoma fragilis (Gmelin 1791) & 29.5 \\
\hline Morum oniscus (Linnaeus 1767) & 2.3 & Modiolus squamosus Beauperthuy 1967 & 38.6 \\
\hline Naticarius canrena (Linnaeus 1758) & 20.5 & Modiolus americanus (Leach 1815) & 4.5 \\
\hline Neodrillia cydia Bartsch 1943 & 4.5 & Moerella guildingii (Hanley, 1844) & 4.5 \\
\hline Neoterebra dislocata (Say 1822) & 6.8 & Musculus lateralis (Say 1822) & 6.8 \\
\hline Nitidella nitida (Lamarck 1822) & 20.5 & Mytilopsis leucophaeata (Conrad 1831) & 4.5 \\
\hline Nitidella sp. Linnaeus 1758 & 2.3 & Saccella acuta (Conrad 1832) & 38.6 \\
\hline Olivella nivea (Gmelin 1791) & 2.3 & Papyridea lata (Born 1778) & 15.9 \\
\hline Phosinella cancellata (Philippi 1847) & 15.9 & Papyridea soleniformis (Bruguière 1789) & 4.5 \\
\hline Phosinella redferni (Espinosa \& Ortea 2002) & 2.3 & Phlyctiderma semiasperum (Philippi 1836) & 25.0 \\
\hline Phosinella sagraiana (d'Orbigny 1842) & 13.6 & Pinctada imbricata Röding 1798 & 61.4 \\
\hline Phrontis alba (Say 1826) & 79.5 & Pitar fulminatus (Menke 1828) & 18.2 \\
\hline Phrontis antillarum (d'Orbigny 1847) & 77.3 & Pitar simpsoni (Dall 1895) & 68.2 \\
\hline Phrontis vibex (Say 1822) & 2.3 & Psammotreta brevifrons (Say 1834) & 9.1 \\
\hline Phyllonotus pomum (Gmelin 1791) & 6.8 & Pteria colymbus (Röding 1798) & 4.5 \\
\hline Pilsbryspira leucocyma (Dall 1884) & 34.1 & Scissula candeana (d'Orbigny 1853 ) & 25.0 \\
\hline Polinices lacteus (Guilding 1834) & 2.3 & Scissula similis (J. Sowerby 1806) & 6.8 \\
\hline Polygona angulata (Röding 1798) & 6.8 & Semele bellastriata (Conrad 1837) & 2.3 \\
\hline Prunum apicinum (Menke 1828) & 88.6 & Semele purpurascens (Gmelin 1791) & 2.3 \\
\hline Prunum carneum (Storer 1837) & 11.4 & Serratina aequistriata (Say 1824) & 11.4 \\
\hline Prunum guttatum (Dillwyn 1817) & 13.6 & Serratina martinicensis (d'Orbigny 1853) & 13.6 \\
\hline Pseudostomatella coccinea (A. Adams 1850) & 4.5 & Solemya occidentalis Deshayes 1857 & 9.1 \\
\hline Pusula pediculus (Linnaeus 1758) & 2.3 & Strigilla carnaria (Linnaeus 1758) & 2.3 \\
\hline Pyrgospira ostrearum (Stearns 1872) & 15.9 & Tagelus divisus (Spengler 1794) & 6.8 \\
\hline Retilaskeya bicolor (C.B. Adams 1845) & 4.5 & Tampaella mera (Say 1838) & 11.4 \\
\hline Rissoina multicostata (C.B. Adams 1850) & 11.4 & Tellina radiata Linnaeus 1758 & 9.1 \\
\hline Schwartziella bryerea (Montagu 1803) & 4.5 & Tellinella listeri (Röding 1798) & 4.5 \\
\hline Schwartziella fischeri (Desjardin 1949) & 6.8 & Trachycardium isocardia (Linnaeus 1758) & 13.6 \\
\hline Smaragdia viridis (Linnaeus 1758) & 22.7 & Trigoniocardia antillarum (d'Orbigny 1853) & 18.2 \\
\hline Strictispira redferni Tippett 2006 & 6.8 & Tucetona pectinata (Gmelin 1791) & 22.7 \\
\hline Strombus pugilis Linnaeus 1758 & 9.1 & Polyplacophora & \\
\hline Tegula fasciata (Born 1778) & 25.0 & Acanthochitona roseojugum Lyons 1988 & 11.4 \\
\hline Turbo cailletii P. Fischer \& Bernardi 1857 & 2.3 & Acanthopleura granulata (Gmelin 1791) & 13.6 \\
\hline Turbo castanea Gmelin 1791 & 2.3 & Chiton tuberculatus Linnaeus 1758 & 9.1 \\
\hline Turritella exoleta (Linnaeus 1758) & 2.3 & Chiton viridis Spengler 1797 & 9.1 \\
\hline Vermicularia knorrii (Deshayes 1843) & 9.1 & Ischnochiton erythronotus (C.B. Adams 1845) & 4.5 \\
\hline Vermicularia spirata (Philippi 1836) & 34.1 & Scaphopoda & \\
\hline Vexillum cubanum Aguayo \& Rehder 1936 & 34.1 & Antalis antillarum (d'Orbigny 1847) & 47.7 \\
\hline Vexillum moniliferum (C.B. Adams 1850) & 9.1 & Antalis cerata (Dall 1881) & 29.5 \\
\hline Volvarina albolineata (d'Orbigny 1842) & 6.8 & Graptacme semistriolata (Guilding 1834) & 6.8 \\
\hline
\end{tabular}

\section{DISCUSSION}

\section{Taxonomic composition of the megazoobenthic community}

The groups to which organisms were assigned comprised upper taxonomic categories that included a high number of species. However, common groups such as ascidians were represented only for one year. Other groups such as isopods, scaphopods, and polychaetes, which have been reported as frequent in the area's benthic community (Alcolado et al. 1998, Claro 2007), were generally found in very low abundance. Amphipods usually present in the region (Ortiz \& Lalana 1996) were not found during this study. This situation suggests that habitat conditions in the area are not good, mainly in BV.

Alcolado et al. (1998) reported 66 Porifera, 140 Mollusca, 100 Crustacea, 90 Polychaeta, 19 Tunicata, and 53 Echinodermata species. Comparatively, species richness at present has decreased by $27.4 \%$. Regarding the same groups (mollusks, crustaceans, polychaetes, echinoderms, ascidians, and poriferans), the total num- 

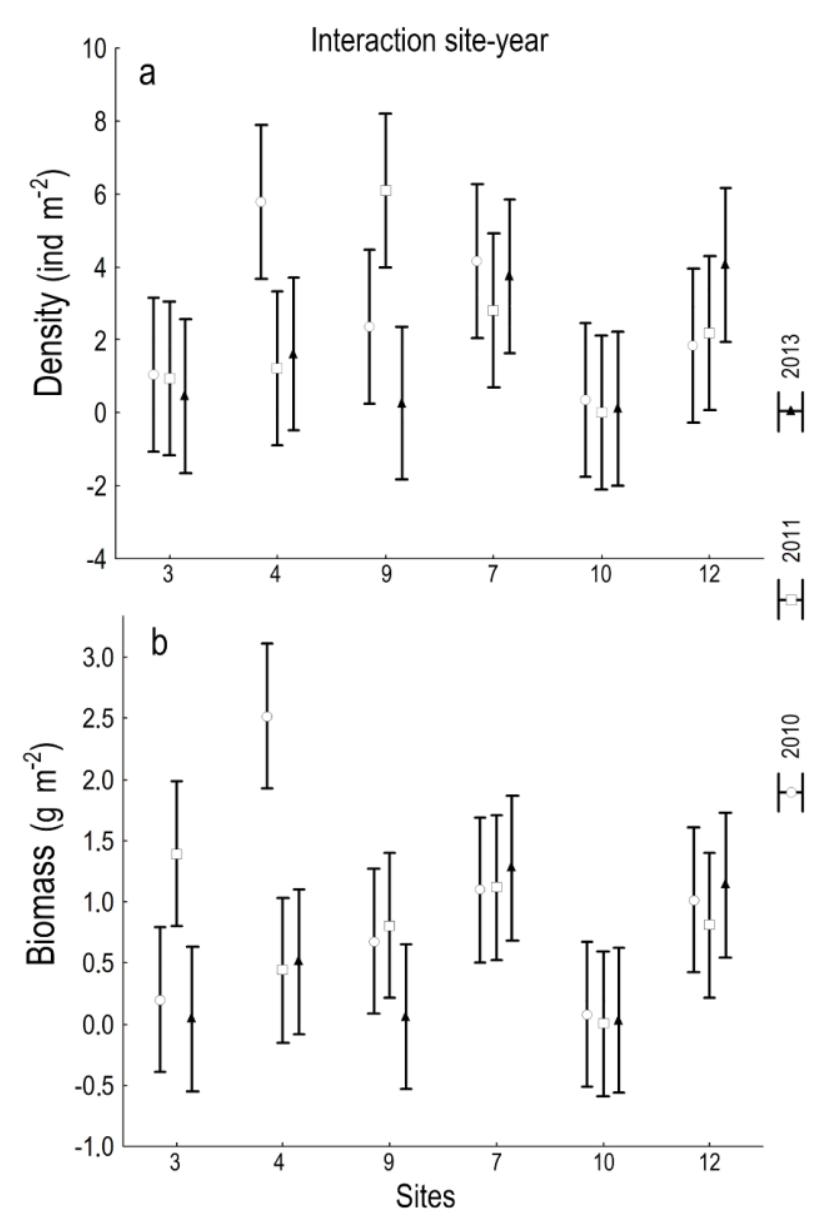

Figure 2. a) Density and b) biomass of soft bottoms megazoobenthic organisms ( $>4 \mathrm{~mm}$ ), found at six routine sites of 2010-2013 period, in San Juan de Los Remedios and Buena Vista bays, Sabana-Camagüey Archipelago, Cuba.

ber of species found in the present work was 22.9 and 43\% lower than the reported by Alcolado et al. (1999) and by Alcolado et al. (2007) respectively. Although we sampled a smaller area in the present study than in previous works, these decrease corroborated softbottom benthic community degradation in the region. Nevertheless, the number of mollusks species was higher than any other reported previously.

The dominance of mollusks in this region has been reported by other authors (Alcolado et al. 1998, 1999, 2007). Mollusks are a fundamental and biodiverse group of the benthic community that comprises species with different feeding habits. Therefore, they play a key trophic role in the ecosystem and intervene in the energy flow and recirculation of matter (MartínezEstalella \& Alcolado 1990, Espinosa et al. 1990). The prevalence of mollusks could be essential for ecosystem sustainability. It could be contributing vitally to the

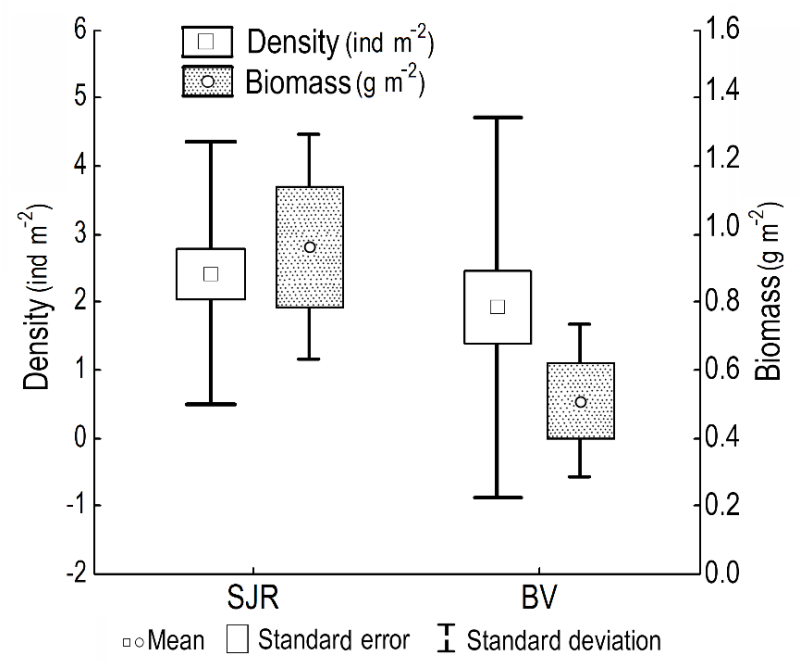

Figure 3. Density and biomass of zoobenthic organisms (>4 mm) found in soft bottoms of San Juan de Los Remedios (SJR) and Buena Vista (BV) bays, SabanaCamagüey Archipelago, Cuba.

slight recovery of the soft-bottom benthic community of this area.

Alcolado et al. (1998) reported that gastropods dominated at external (toward platform edge) sites. In the present study, gastropods' dominance at any site was considered a mollusk community response to the region's physiographic conditions and environmental stress levels; most recorded mollusk species coincided with those reported by Alcolado et al. (1998), described as lobsters' natural diet components (Herrera et al. 1991, Martínez-Coello et al. 2015). The recorded species richness per site $(S=17)$ was similar to that reported by Alcolado et al. (1998). This evidence suggests that the main changes in the benthic community regard the abundance and biomass of organisms.

In the past, it was reported that represented groups and dominant species varied considerably between locations because of the high ecological heterogeneity in this region, making evident ecological succession processes and the coexistence of opportunistic species with similar requirements and wide physiological tolerance (Jiménez \& Ibarzábal 1982, Alcolado et al. 1998). Although the anthropogenic influence should have introduced a relatively homogenous impacted condition in all this region, some remained heterogeneity characteristics that could explain variations among sites and lends great importance to the similarities that could be found.

The species Chione cancellata was reported among the most abundant organisms in the SCA region's interior sites, associated with muddy, but not too muddy or liquid (silt and clay) sediment. This species 
Table 4. Average density and biomass of benthic organisms ( $>4 \mathrm{~mm}$ ) of soft bottoms inside and outside the Zone Under Special Regime of Use and Protection in Buena Vista Bay between 2011 and 2017. n: number of individuals, $t$ : Student's $t$-test value, df: degrees of freedom, SE: standard error, SD: standard deviation, ${ }^{*} P$-values $(>0.05)$.

\begin{tabular}{|c|c|c|c|c|c|c|c|c|}
\hline \multicolumn{9}{|c|}{2011} \\
\hline & $\mathrm{n}$ & Inside & Outside & $t$ & $\mathrm{df}$ & $\mathrm{SE}$ & SD & $P$ \\
\hline Density (ind $\mathrm{m}^{-2}$ ) & 6 & 3.05 & 2.34 & 0.33 & 10 & 5.18 & 0.63 & 0.747 \\
\hline Biomass $\left(\mathrm{g} \mathrm{m}^{-2}\right)$ & 6 & 0.40 & 0.98 & -1.75 & 10 & 0.69 & 0.41 & 0.111 \\
\hline \multicolumn{9}{|c|}{2017} \\
\hline Density (ind $\mathrm{m}^{-2}$ ) & 6 & 1.61 & 10.83 & -3.09 & 10 & 1.74 & 7.10 & $0.011 *$ \\
\hline Biomass $\left(\mathrm{g} \mathrm{m}^{-2}\right)$ & 6 & 0.80 & 2.35 & -3.47 & 10 & 0.89 & 0.64 & $0.006^{*}$ \\
\hline
\end{tabular}

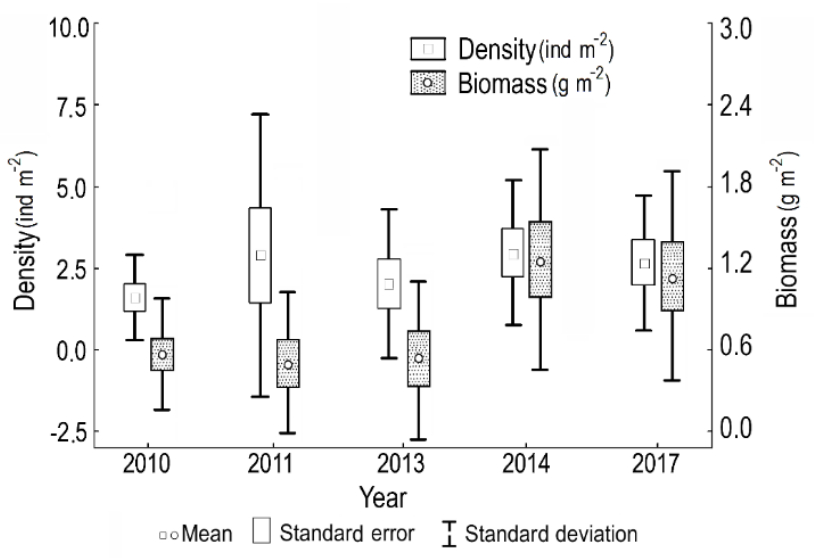

Figure 4. Annual variation of the density and biomass of zoobenthic organisms (>4 $\mathrm{mm}$ ) in the soft bottoms of the Buena Vista, Bay, Cuba (sites 9, 10, and 12).

lives on the bottom surface, but it can not survive as deeper as other bivalves like Lucinisca muricata (Alcolado et al. 1998, Bitter et al. 2009). In the present study, C. cancellata was found mostly at sites 1 and 3, suggesting muddy sediments at both sites, where marine vegetation was also scarce or absent. Even though this species was frequently recorded, densities were considerably lower than those reported by Prieto et al. (1998) at Cariaco Gulf, a good quality marine area of Venezuela where this species is cultivated.

In the studied region, the abundance of ophiuroids has been related to higher wave activity and sediment resuspension (Alcolado et al. 1998). Ophiuroids were abundant at sites $9,11,16$, and 18 , associated with the presence of red and brown algae, which have been reported for this area (Martínez-Daranas et al. 2009; Martínez-Daranas \& Suárez 2018). The species Ophiothrix angulate has been reported as the most common echinoderm in other Caribbean countries' bays, in soft sand-mud bottom colonized by sponges and sandy bottoms with seagrass Thalassia testudinum, macroalgae, and sponges (Quirós-Rodríguez 2015).

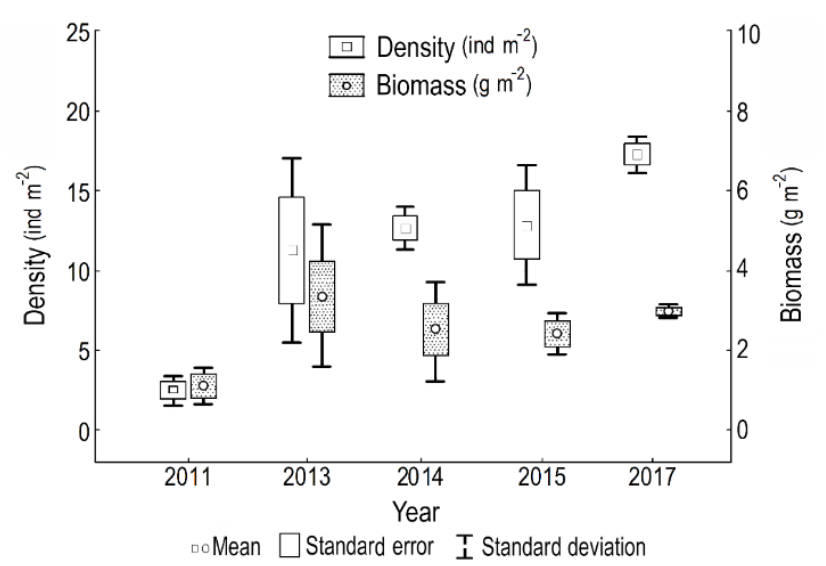

Figure 5. Annual variation of zoobenthic organisms' density and biomass ( $>4 \mathrm{~mm}$ ) at site 11 , Buena Vista Bay, Sabana-Camagüey Archipelago, Cuba.

All studies worldwide and in the SCA region conclude that benthic communities are better developed in the presence of seagrasses (Martínez-Daranas et al. 2009, Martínez-Daranas \& Suárez 2018, Munroe et al. 2018). The present study corroborated that crustaceans were better represented on substrates with some vegetable cover. At the same, numerous specimens of the green sea urchin, Lytechinus variegatus were collected at sites with the presence of seagrass. This species is considered an important herbivore in seagrasses and is fundamental for energy transfer (Corvea et al. 1990, Arias-Schreiber et al. 2008). The values of total biomass and density were slightly higher at sites where the number of these sea urchins was higher.

\section{Megazoobenthos density and biomass. Comparison between bays and between protected and non- protected areas}

Total density and biomass during the 2010-2013 period were low and stable in both bays, with no differences between years indicating that environmental stress and 
anthropogenic impact suggested by Alcolado et al. (1998) still prevail. Except for site 11, there were no statistical differences among sites sampled each year (from 2010 to 2013), indicating a similar (homogenous) and impacted condition for benthos in this region. Based on these analyses for the 2010-2013 period (made for GEF/PNUD Project), the hypothesis of an improvement in the area was not confirmed. However, posterior samples showed that the density of organisms at site 11 significantly increased between 2011 and 2017. Biomass recorded in 2017 was also considerably higher than in 2011. Hence, it may be possible that recovery at a micro-area (site) could be detected. Species richness values, density, and biomass of organisms found at this site during 2015 and 2017 were significantly higher than those reported by Alcolado et al. (1998), which could be related to the positive effect of management measures such as the banning of trawling, which had a lower intensity at BV than at JSR (Claro et al. 2004). It could also be linked to the protection measures increase in the area, considering that site 11 was located within the ZUSRUP.

Although between 2010-2013, total biomass increased at site 7 (SJR) and total density at site 12 (BV), both located within the ZUSRUP. Samples taken between 2014 and 2017 showed that these trends were not consistent because there was not enough data (to analyze the situation at site 7 after 2013). There were no significant differences between 2010 and 2017 (site 12). Nevertheless, sites 9 and 10, which had low abundance and biomass, even showing a trend towards a decrease, were located in BV, outside the limits of the ZUSRUP. Total density and biomass of organisms were similar inside and outside the ZUSRUP at the beginning of the study (2011). Still, they were both significantly higher inside the ZUSRUP in 2017 (at the end of the study). These facts may suggest a potential positive effect of creating protected areas.

Areas with greater oceanic exchange and lower anthropogenic influence (further away from the coast) presented higher density and diversity of organisms, which corroborates other studies results in the region, that reported a greater abundance of seagrasses and associated fauna at that edge sites (Martínez-Daranas et al. 2009, Martínez-Daranas \& Suárez 2018). Sites that presented lower density and species richness values were located toward the bays' interior (site 10 in BV and site 3 in SJR). Regarding biomass, sites with lower values (sites 10 and 9) were also located in the interior, both in BV. According to Alcolado et al. (1998), the factors that most affect the benthic fauna in this region include the elevated and fluctuating salinity, high organic matter content in sediment, too muddy substrates, and lack of microphytobenthos.
Since the past century, relatively high nickel levels associated with anthropogenic influence have been reported in sediments of SJR and BV (Martínez-Canals et al. 1998). Additionally, Montalvo et al. (2004) described hypoxic conditions in BV and argued low water quality according to Cuban norm NC-25:1999, based on elevated $\mathrm{DBO}_{5}$ and ammonium values. Phosphorus concentrations, mainly in the interior of SJR and BV, have been reported as the highest of all values recorded for SCA. These bays' interior waters have been classified as between oligotrophic and eutrophic (Montalvo et al. 2004). Moreover, bottom trawling's past activity was more intense in the interior, which increased deterioration and stressful conditions to which these areas were exposed (Quirós 2006). More recent studies corroborated hypoxic conditions in the two bays and detected high organic matter levels in the entire SCA's interior waters (Montalvo et al. 2008). Sites 13 and 14, located near Cayo Santa Maria, presented the lowest densities and biomass recorded in 2015. Cayo Santa Maria is located at the northern limit of $\mathrm{BV}$, and it is exploited for tourism having relatively high anthropogenic activity levels.

Before implementing resolutions banning seine nets and trawling, these fishing types did not occur with the same intensity in all sampled areas. Also, banning bottom trawl fisheries did not take place at the same time in all sampled sites. This situation could contribute to explain the differences found between the sampled sites. At Boca Chica, an area very close to site 4 (SJR), it was reported that seagrass cover showed a decrease of approximately 50\% between 1994 and 2002. This decrease was attributed, essentially, to the use of seine nets and variations in salinity (Quirós 2006).

No evidence of recovery was detected in the benthic communities at any site based only on data recorded during 2010-2013. However, the combined analysis of subsequent sampling (until June 2017) provided the first evidence that suggested an incipient recovery inside the ZUSRUP, which is mainly based on the greater values of total density and biomass at site 11 and the decreasing, or not increments, at sites like 9 and 10, located outside the ZUSRUP. On the other hand, higher values of biomass and density observed in SJR, where seine net activity was more intense than in BV (Claro et al. 2004), could be due to the banned trawling fishing, suggesting the positive effects of this regulatory. Nevertheless, such higher density and biomass values could also be related to a greater recovery of biological communities in JSR's external sites, with greater water exchange. Several authors reported greater turbidity and salinity levels in the western BV sector, limiting the seagrasses development, inducing low water quality, and affecting benthic 
communities, decreasing potential food sources for organisms such as the spiny lobster Panulirus argus (Fernández-Vila \& Chirino-Núñez 1993, BetanzosVega et al. 2013). Lobster catches in this region (Caibarien) had decreased from $700 \mathrm{t}$ (1985-1989) to 120 t (2010-2014), which means $82.86 \%$. Nowadays, lobster catches annually by the EPICAI fishery industry from the Caibarien region remain low, but with a little increase compared to those in the 2010-2014 period, with $140.7 \mathrm{t}$ in 2018 and $151.5 \mathrm{t}$ in 2019 (AlzugarayMartínez comm. pers.).

\section{CONCLUSIONS}

The megazoobentonic communities of San Juan de Los Remedios Bay and Buena Vista Bay are evidence of an affected habitat. Nevertheless, mean values of density and biomass, and the number of represented species, were lower in Buena Vista, suggesting more impacted communities and lesser recovery in this bay.

There were not numerous statistical differences among sites sampled each year, which indicates a homogenous condition for benthos in this region. However, sites near to the coast or the causeway showed lesser values. Temporary variations of the megazoobenthic biomass and density showed an increment of the differences inside and outside the Zone Under Special Regime of Use and Protection. Higher average values among sites inside the protected area for the 2017 year could suggest an incipient recovery and the potential effectiveness of this management measure.

Greater values of biomass, density, and diversity of organisms in San Juan de Los Remedios Bay, where seine net activity was more intense than in Buena Vista Bay, suggest an incipient recovery after banned bottom trawl fisheries.

\section{ACKNOWLEDGMENTS}

ALC thanks CONACYT for a doctoral fellowship. We are also grateful to the Fisheries Research Center of Cuba and fishers and managers of the Caibarien (EPICAI fishery industry) who collaborate in the fieldwork. The help of José Espinosa Sáez's organism identification was of immeasurable value. LAAC is grateful for the support received through COFAA and EDI from the Instituto Politécnico Nacional.

\section{REFERENCES}

Alcolado, P.M. \& Espinosa, J. 1996. Empleo de las comunidades de moluscos marinos de fondos blandos como bioindicadores de la diversidad del megazoobentos y de la calidad ambiental. Iberus, 14: 79-84.

Alcolado, P.M., García, E.E. \& Arellano-Acosta, M.E. 2007. Ecosistema Sabana-Camagüey. Estado actual, avances y desafíos en la protección y uso sostenible de la biodiversidad. Editorial Academia, La Habana.

Alcolado, P.M., García, E.E. \& Espinosa, N. 1999. Protecting biodiversity and establishing sustainable development in the Sabana-Camaguey ecosystem. Serie GEF/PNUD, Proyecto Sabana-Camaguey CUB/ 92/G31. Instituto de Oceanología, La Habana.

Alcolado, P.M., Espinosa, J., Martínez-Estalella, N., lbarzábal, D., del Valle, R., Martínez-Iglesias, J.C., et al. 1998. Prospección del megazoobentos de los fondos blandos del Archipiélago Sabana-Camagüey, Cuba. Avicennia, 8/9: 87-104.

Arias-Schreiber, M., Wolff, M., Cano, M., MartínezDaranas, B., Marcos, Z., Hidalgo, G., et al. 2008. Changes in benthic assemblages of the Gulf of Batabanó (Cuba) results from cruises undertaken during 1981-85 and 2003-04. Pan-American Journal of Aquatic Science, 3: 49-60.

Betanzos-Vega, A., Siam-Lahera, C. \& ArencibiaCarballo, G. 2010. Variación de la salinidad y su relación con la distribución por talla del ostión de mangle en Villa Clara, Cuba. Revista Cubana de Investigaciones Pesqueras, 27: 41-46.

Betanzos-Vega, A., Capetillo-Piñar, N., LopezteguiCastillo, A. \& Martínez-Daranas, B. 2013. Variación espacio-temporal de la turbidez y calidad en cuerpos de agua marina de uso pesquero, región norcentral de Cuba, 2008-2010. Serie Oceanológica, 12: 24-35.

Bitter, R., Didonna, G. \& Viéitez, J.M. 2009. Caracterización de la comunidad de moluscos asociada a Thalassia testudinum en localidades del Parque Nacional Mocorroy, Venezuela. Ciencia, 17: 151-168.

Castellanos-Romero, K., Pizarro del Río, J., CuentasVillarreal, K., Costa-Anillo, J.C., Pino-Zarate, Z., Gutierrez, L.C., et al. 2017. Lentic water quality characterization using macroinvertebrates as bioindicators: an adapted BMWP index. Ecological Indicators, 72: 53-66.

Chirwa, E.R. \& Chilima, L. 2017. Use of benthic macroinvertebrate indices as bioindicators of ecosystem health for the detection of degraded landscapes in Malawi. International Journal of Agriculture, Forestry and Fisheries, 5: 128-134.

Çinar, M.E., Bakır, K., Öztürk, B., Katağan, T., Dağl1, E., Açık, Ş., et al. 2015. TUBI (Turkish Benthic Index): a new biotic index for assessing impacts of organic pollution on benthic communities. Journal of the Black Sea/Mediterranean Environment, 21: 135-168. 
Claro, R. 2007. La biodiversidad marina de Cuba. (CDROM), Instituto de Oceanología, Ministerio de Ciencia, Tecnología y Medio Ambiente, La Habana, Cuba. [http://repositorio.geotech.cu/jspui/handle/1234/ 1397]. Reviewed: November 15, 2018.

Claro, R., García-Arteaga, J.P., Gobert, B., CantelarRamos, K., Valle-Gómez, S.V. \& Pina-Amargós, F. 2004. Situación actual de los recursos pesqueros del archipiélago Sabana-Camagüey, Cuba. Boletín de Investigaciones Marinas y Costeras, 33: 49-67.

Corvea, A., Abreu, M. \& Alcolado, P.M. 1990. Características de las comunidades de equinodermos de la macrolaguna del Golfo de Batabanó. In: Alcolado, P.M. (Ed.). El bentos de la macrolaguna del Golfo de Batabanó. Editorial Academia, La Habana, pp. 90-99.

Cruz, R., Baisre, J.A., Díaz, E., Brito, R., Blanco, W., García, C. \& Carrodeguas, C. 1990. Atlas biológicopesquero de la langosta en el archipiélago Cubano. Revista Cubana de Investigaciones Pesqueras y Revista Mar y Pesca (número especial), 125 pp.

Del Valle, R., Solís-Marín, F.A., Abreu-Pérez, M., LaguardaFigueras, A. \& Durán-González, A. 2005. Catálogo de los equinodermos (Echinodermata: Crinoidea, Echinoidea, Holothuroidea) nerítico-bentónicos del Archipiélago Cubano. Revista de Biología Tropical, 53: 928.

Espinosa, J., Herrera, A., Brito, R., Díaz-Iglesias, E., González, G., Ibarzábal, D. \& Gotera, G. 1990. Los moluscos en la dieta de la langosta del Caribe Panulirus argus (Crustacea: Decapoda). Iberus, 9: 127-140.

Fernández-Vila, L.J. \& Chirino-Núñez, A.L. 1993. Atlas oceanográfico de las aguas del Archipiélago de Sabana-Camagüey. Instituto Cubano de Hidrografía, La Habana.

Fouad, M., Bessi, H., Benhra, A. \& Bouhallaoui, M. 2019. The benthic community used as bioindicator for assessment of the quality of a Moroccan coastal ecosystem: Moulay Bousselham Lagoon. International Journal of Engineering Research \& Technology, 8: 127-13.

Gómez, O., Ibarzábal, D. \& Silva, A. 1980. Evaluación cuantitativa de bentos en la región Suroccidental de Cuba. Oceanology Institute Technical Report, 149: 125.

Herrera, A., Díaz-Iglesias, E., Brito, R., González, G., Gotera, G., Espinosa, J. \& Ibarzábal, D. 1991. Alimentación natural de la langosta Panulirus argus en la región de los Indios (Plataforma SW de Cuba) y su relación con el bentos. Revista de Investigaciones Marinas, 12: 172-182.
Ibarzábal, D., Del Valle, R., Abreu, M., Espinosa, J. \& Cortés, R. 1999. Listado preliminar de los poliquetos, moluscos y equinodermos marinos del Archipiélago de los Jardines de la Reina. Cocuyo, 9: 3-6.

Jiménez, C. \& Ibarzábal, D. 1982. Evaluación cuantitativa del mesobentos en la plataforma nororiental de Cuba. Ciencias Biológicas, 7: 53-70.

Lalana, R. \& Ortiz, M. 2000. Lista actualizada de los crustáceos decápodos de Cuba. Revista de Investigaciones Marinas, 21: 33-44.

Lalana, R., Capetillo, N., Brito, R., Díaz, E. \& Cruz, R. 1989. Estudio del zoobentos asociado a Laurencia intricada en un área de juveniles de langosta, al SE de la Isla de la Juventud, Cuba. Revista de Investigaciones Marinas, 10: 207-217.

Martínez-Canals, M., Pérez, R., Rodríguez, A. \& Lorente, Y. 1998. Nivel de contaminación metálica de los sedimentos de fondo de algunas zonas de la plataforma insular cubana. Contribución a la Educación y la Protección Ambiental, 1: 65-69.

Martínez-Coello, D., Lopeztegui, A. \& Amador, U. 2015. Diferencias entre sexos en la composición de la dieta natural de la langosta Panulirus argus (Decapoda: Palinuridae) al este del golfo de Batabanó, Cuba. Cuadernos de Investigación UNED, 7: 269-277.

Martínez-Daranas, B., Cano-Mallo, M. \& Clero-Alonso, L. 2009. Los pastos marinos de Cuba: estado de conservación y manejo. Serie Oceanológica, 5: 24-44.

Martínez-Daranas, B. \& Suárez, A.M. 2018. An overview of Cuban seagrasses. Bulletin of Marine Science, 94: 269-282. doi: 10.5343/bms.2017.1014

Martínez-Estalella, N. \& Alcolado, P.M. 1990. Características generales de las comunidades de moluscos de la macrolaguna del Golfo de Batabanó. In: Alcolado, P.M. (Ed.). El bentos de la macrolaguna del Golfo de Batabanó. Editorial Academia, La Habana, pp. 53-74.

Matangulu, M., Gurung, S., Prajapati, M. \& Jyakhwo, R. 2017. Macroinvertebrate assemblages as indicators of water quality of the West Seti river, Bajhang, Nepal. International Journal of Environment, 6: 25-45.

McGovern, M., Poste, A.E., Oug, E., Renaud, P.E. \& Trannum, H.C. 2020. Riverine impacts on benthic biodiversity and functional traits: a comparison of two sub-Arctic fjords. Estuarine, Coastal and Shelf Science, 240: 106774.

Middelburg, J.J. 2018. Reviews and syntheses: to the bottom of carbon processing at the seafloor. Biogeosciences, 15: 413-427. doi: 10.5194/bg-15-413-2018

Mikkelsen, P.M. \& Bieler, R. 2007. Seashells of Southern Florida. Living marine mollusks of the Florida Keys and adjacent regions: bivalves. Princeton University Press, Princeton.

Mohammad-Karami, A., Riyahi-Bakhtiari, A., Kazemi, A. \& Kheirabadi, N. 2014. Assessment of toxic metals 
concentration using pearl oyster, Pinctada radiate, as bioindicator on the coast of Persian Gulf, Iran. Iranian Journal of Toxicology, 7: 956-961.

Montalvo, J.F., García, I., Perigó, E., Martínez, M. \& Cano, M. 2004. Niveles más representativos de los parámetros de calidad químico-ambiental en la ecorregión Sabana-Camaguey. Contribución a la Educación y a la Protección Ambiental, 5: 58-70.

Montalvo, J.F., García, I., Loza, S., Esponda, S.C., César, M.E., González de Zaya, R. \& Hernández, L. 2008. Oxígeno disuelto y materia orgánica en cuerpos de aguas interiores del Archipiélago Sabana-Camagüey, Cuba. Serie Oceanológica, 4: 71-84.

Munroe, S.E.M., Coates-Marnane, J., Burford, M.A. \& Fry, B. 2018. A benthic bioindicator reveals distinct land and ocean-based influences in an urbanized coastal embayment. Plos One, 13: e0205408. doi: 10.1371/ journal.pone. 0205408

Ortiz, M. \& Lalana, R. 1996. Los anfípodos de la primera expedición conjunta CUBA-USA, a bordo del B/I "ULISES", a las aguas del Archipiélago SabanaCamaguiey, Cuba, en 1994. Anales Instituto de Biología UNAM, Serie Zoológica, 67: 89-101.

Ortiz, M. \& Lalana, R. 2001. Guía ilustrada para la identificación de los estomatópodos (Crustacea, Hoplocarida), del archipiélago cubano. Revista de Investigaciones Marinas, 22: 179-186.

Ovaskainen, O., Weigela, B., Potyutkoc, O. \& Buyvolovc, Y. 2019. Long-term shifts in water quality show scaledependent bioindicator responses across RussiaInsights from 40 year-long bioindicator monitoring program. Ecological Indicators, 98: 476-482. doi: 10.1016/j.ecolind.2018.11.027

Prieto, A.S., Ramos, C. \& Arrieche, D. 1998. Producción secundaria de una población de Chione cancellata (Bivalvia: Veneridae) de la costa sur del Golfo de Cariaco, Venezuela. Revista de Biología Tropical, 46: 913-919.

Received: 20 April 2020; Accepted: 28 September 2020
Prieto, A.S., Sant, S., Méndez, E. \& Lodeiros, C. 2003. Diversidad y abundancia de moluscos en las praderas de Thalassia testudinum de la Bahía de Mochima, Parque Nacional Mochima, Venezuela. Revista de Biología Tropical, 51: 413-426.

Puga, R., Valle, S., Kritzer, J.P., Delgado, G., De León M.E., Giménez, E., et al. 2018. Vulnerability of nearshore tropical finfish in Cuba: implications for scientific and management planning. Bulletin of Marine Science, 94: 1-16.

Quirós, A. 2006. Bases gnoseológicas del uso sostenible de los recursos marino-costeros de Villa Clara. CESAM-CITMA, Villa Clara. [http://www.forumcyt.cu/UserFiles/forum/Textos/0500487.pdf]. Reviewed: November 29, 2019.

Quirós-Rodríguez, J.A. 2015. Echinoderms in shallowbottom from Ahumadera Sector, Cispatá Bay, Cordoba, Colombian Caribbean. Acta Biológica Colombiana, 20: 101-108. doi: 10.15446/ abc.v20n1. 42529

Redfern, C. 2013. Bahamian seashells: 1161 species from Abaco, Bahamas. Bahamian Seashells, Florida.

Tlili, S. \& Mouneyrac, C. 2019. The wedge clam Donax trunculus as sentinel organism for Mediterranean coastal monitoring in a global change context. Regional Environmental Changes, 19: 995-1007. doi: 10.1007/s10113-018-1449-9

Warmke, G.L. \& Abbott, R.T. 1975. Caribbean seashell: a guide to the marine mollusks of Puerto Rico and other West Indian Islands, Bermuda and the lower Florida keys. Dover Publication, New York. 